\title{
EL CUADRADO DE OPOSICIÓN COMO INSTRUMENTO DE LA LÓGICA: SU USO Y APLICACIONES EN TOMÁs DE MERCADO
}

\author{
Juan Manuel Campos Benítez \\ Benemérita Universidad Autónoma de Puebla \\ juancamposb@hotmail.com
}

\begin{abstract}
Logic, as a knowledge instrument, was developed since and during the preaching of the Gospel in our American lands. The New Spain authors make use of the square of opposition — which they spread to unsuspected pointsin order to introduce their logic doctrine. In this work I will show some of the square uses in the temporal and modal logic, and Tomás de Mercado's quantified modality. The square's logical doctrine and its uses can be compared to nowadays logic's extensions.

Key Words: square of opposition, Tomás de Mercado, modal logic, temporal logic.
\end{abstract}

\section{Resumen}

La lógica como instrumento del pensamiento fue cultivada en nuestras tierras americanas desde los inicios de la evangelización y a la par de ella. Los autores novohispanos presentan su doctrina lógica haciendo uso del cuadrado de oposición, el cual expanden hasta extremos insospechados. En este artículo quiero mostrar algunos usos del cuadrado para la lógica temporal, la lógica modal y la modalidad cuantificada en Tomás de Mercado. La doctrina lógica contenida en el cuadrado y sus aplicaciones bien puede compararse con lo que en nuestros días estudian las llamadas "extensiones" de la lógica.

Palabras clave: cuadro de oposición, Tomás de Mercado, lógica modal, lógica temporal.

\footnotetext{
*Recibido: 04-09-07. Aceptado: 23-11-07.
} 


\section{El cuadrado temporal de Tomás de Mercado}

\subsection{El cuadrado tradicional}

El cuadrado tradicional consta de cuatro tipos o formas de oraciones a las que se les ha asignado una vocal y son estas: $A$ : universal afirmativa; $E$ : universal negativa; $I$ : particular afirmativa y $O$ : particular negativa. Si tomamos una oración cuantificada de la forma Sujeto-cópula-Predicado tenemos cuatro combinaciones ordenadas en un cuadro (ver figura 1).

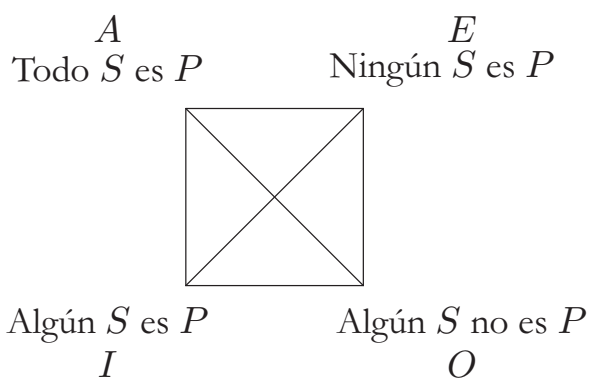

Figura 1: Cuadro de oposición.

Las de arriba $(A-E)$ son universales y son contrarias; las de abajo $(I-O)$ son particulares y son subcontrarias; las del lado izquierdo $(A-I)$ son afirmativas y subalternas; las del lado derecho $(E-O)$ son negativas y subalternas; los extremos diagonales son contradictorias $(A-O, E-I)$. Cada una tiene formas equivalentes, pues, por ejemplo decir que "todo $S$ es $P$ " es equivalente a "no es el caso que algún $S$ no sea $P$ ".

Los términos que aparecen en la oración pueden ser singulares (como "Pedro") o comunes (como "hombre"); los términos comunes admiten cuantificación. Los términos sujeto y predicado de nuestras oraciones — y de la silogística — son términos comunes y pueden cuantificarse universal y particularmente. Pues bien, los medievales decían que cuando un término está cuantificado tiene "suposición" (es decir, su alcance referencial); los términos cuantificados universalmente tienen suposición 
distributiva y los términos cuantificados particularmente tienen suposición determinada; esta terminología nos será útil para entender a Mercado ${ }^{1}$. Una expresión como "todo" en "todo hombre" se refiere a éste, y a ése, a aquél, a cada uno, está "distribuido" por decirlo así; pero "algún hombre" se refiere a éste, o a ése, o a aquél. Podemos decir que el cuantificador se resuelve o analiza de esa manera. Claro que en nuestro cuadrado habría que analizar la suposición de toda la oración, es decir, tanto del sujeto como del predicado, pues para el medieval ambos están cuantificados; para nuestros fines nos basta con lo que hemos dicho. La cópula es el verbo ser, que aparece en nuestro esquema " $S$ es $P$ ". Admite distinciones: modales y temporales, pues tendremos oraciones como "algún hombre posiblemente disputa", "Pedro fue docto" y otras. Trataremos aquí las modales y temporales expresadas en los cuadrados de oposición.

\subsection{Los tiempos y la cuantificación}

La oración de tiempo presente se llama oración "asertórica” o de “inherencia", de inesse o de tiempo "intrínseco". Las oraciones temporales son de tiempo extrínseco, ya sean de pasado o de futuro. Estas oraciones de inherencia también se distinguen de las modales. Las oraciones modales están calificadas por un modo (una expresión como "posible", "necesario") pero las no modales carecen de modo y estrictamente hablando son de tiempo presente. Cada tiempo puede expresarse en un cuadrado.

Mercado no presenta un cuadrado temporal de oposición y equivalencia para los tiempos, pero puede reconstruirse siguiendo sus instrucciones. En efecto, en varias partes hace alusiones al tiempo y su comportamiento lógico relacionándolo con la doctrina de la suposición de los términos.

\footnotetext{
${ }^{1}$ En rigor habría que decir: el término "sujeto", por oposición al término "predicado". Cuando el término sujeto no estaba cuantificado, el caso del término singular, le llamaban suposición discreta.
} 
Así como los tiempos son tres, a saber, presente, pretérito y futuro, así también la cópula es triple. Pero ciertamente en los tiempos hay una distinción amplia. Pues el tiempo presente es indivisible; pero el pretérito y el futuro tienen extensión y partes. Por lo cual, las cópulas extrínsecas "fue" y "será", ya que importan estas diferencias de tiempo, son resolubles. Al modo como (digo) "hombre" tiene diversos supuestos en los que se resuelve, así "fue" tiene también diversas partes de tiempo en las que puede resolverse, y por las cuales supone, ahora determinadamente, ahora distributivamente ${ }^{2}$.

Si el pasado y el futuro tienen "partes", pueden cuantificarse y la cuantificación será universal (distributiva) y particular (determinada), como dice Mercado. Así como "hombre" cuantificado universalmente se refiere a cada hombre y cuantificado particularmente se refiere por lo menos a alguno, así también los tiempos pretérito y futuro han de referir a todo tiempo o a algún tiempo. La referencia será a momentos, instantes, intervalos según lo queramos interpretar y de acuerdo a lo que queremos estudiar; ${ }^{3}$ no entraremos en estos aspectos.

Podemos considerar "fue" y "será" como operadores temporales determinados, es decir, particulares. Ahora bien, así como al negar una oración particular obtenemos una oración universal negativa, dado que la contradictoria de la cuantificación particular afirmativa es la universal negativa, así también ocurre con los operadores temporales.

Sea la oración "Pedro disputa" calificada temporalmente. Simbolizando esa oración con una constante individual ( $p$ : Pedro) y una constante predicativa $(D$ : disputa) tenemos:

\section{$D p$}

\footnotetext{
${ }^{2}$ Cfr. Tomás de Mercado: Comentarios lucidísimos al texto de Pedro Hispano, trad. M. Beuchot, México: UNAM 1986, p. 287. Todas nuestras citas provienen de aquí, en adelante citaré la página entre paréntesis en el cuerpo del texto.

${ }^{3}$ Por ejemplo "Pedro golpeó a Pablo" o "Pedro estornudó" admiten interpretación según instantes, pero "Pedro convenció a Pablo" o "Pedro enfermó" admiten intervalos.
} 
a la cual podemos añadir el operador temporal "P", pasado:

$$
\mathbf{P} D p
$$

y que puede leerse también como en éstas:

"En algún momento del pasado Pedro disputa".

$$
\text { “alguna vez Pedro disputó”. }
$$

Si la negamos

$$
\neg \mathbf{P} D p
$$

queremos decir que nunca disputó, pues la negación de "alguna vez Pedro disputó" es "no es el caso que alguna vez...", es decir, nunca lo hizo. Pongo otro ejemplo: si afirmo que Sócrates no fue sabio, es decir, niego la cópula temporal determinada, estoy afirmando que en cada momento de su vida Sócrates no tuvo la propiedad de ser sabio. Así que la oración "Sócrates no fue sabio" se resuelve o analiza como

"Para cada instante del pasado (durante la vida de Sócrates), 'Sócrates no es sabio' es verdadera".

Claro que se trata de una oración falsa, pues si bien es cierto que en algún momento no fue sabio, en otros momentos de su vida sí lo fue.

Tenemos pues que "fue" y "será" son particulares y estarán en la parte inferior del cuadrado; sus contradictorias serán universales y corresponden a la parte superior, como veremos. Lo mismo pasa cuando al operador temporal le sigue una negación, tendremos la oración tipo $O$. Mercado observa esto cuando dice que de una oración como:

$$
\text { "Sócrates fue no sabio", }
$$

resulta inválido concluir de ahí que:

$$
\text { "Sócrates no fue sabio", }
$$


ya que la primera tiene suposición determinada y de ahí no se puede concluir una suposición distributiva; el lector ya estará al tanto y podrá asentir con fray Tomás al entender que de una particular negativa no se sigue una universal negativa. Pero vayamos a las condiciones de verdad de las oraciones temporales y a los cuadrados de oposición para el pasado y futuro.

\subsection{Condiciones de verdad y cuadrados temporales}

Dice Mercado:

Para la verdad de la proposición de pretérito se requiere que su proposición de inherencia alguna vez haya sido verdadera. Se entiende en la afirmativa, porque en la negativa se requiere que siempre haya sido verdadera... Pero, para la verdad de la proposición de futuro se requiere que tenga después de ahora una proposición de inherencia que alguna vez sea verdadera (p. 285).

Note el lector las expresiones "alguna vez" y "siempre"; sugieren que los operadores temporales $P$ y $F$ admiten interpretación según los cuantificadores. Podemos reconstruir dos cuadrados temporales, uno para el pasado y otro para el futuro. Primero un cuadro "general": en lugar de $I$ tendremos "Alguna vez"; en lugar de $E$ tendremos "No es el caso que alguna vez", que equivale a "Nunca"; en lugar de $O$ tendremos "Alguna vez no". $A$ es la negación de $O$ : "No alguna vez no", que equivale a "Siempre". Lo mismo valdrá para los operadores del pasado y del futuro, en cuyos casos la universal afirmativa debe leerse "Siempre (en el pasado/en el futuro)" y la universal negativa "Nunca (en el pasado/en el futuro)". 


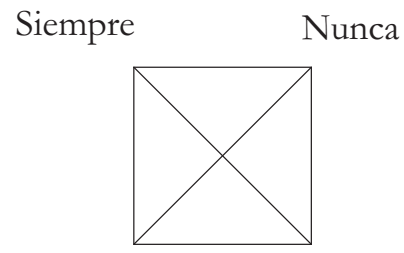

Alguna vez Alguna vez no

Figura 2: Cuadro temporal.

Podemos combinar ambos cuadros, donde la línea vertical representa el presente. Las relaciones lógicas de estos cuadrados son las mismas que las del cuadrado tradicional, así que pueden obtenerse un número considerable de teoremas temporales ${ }^{4}$.

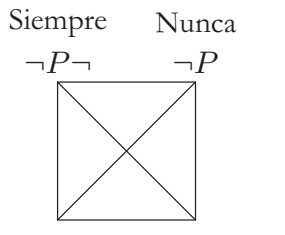

Alguna vez Alguna vez no

$P$

$P \neg$

Siempre Nunca

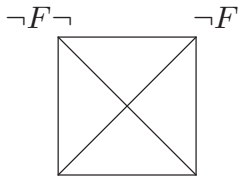

Alguna vez Alguna vez no

$F \quad F \neg$

Pasemos ahora a la modalidad.

\footnotetext{
${ }^{4}$ Actualmente se usa $H$ para "siempre en el pasado" y $G$ para "siempre en el futuro". También puede expresarse esto en términos de cuantificación: $(t) p^{t}$ : "la proposición $p$ es verdadera en todo instante $t$ ", si $t$ es menor a cero expresaremos el pasado, si es mayor a cero el futuro. Si es igual a cero expresa el presente, pero no hace falta entonces el indicador temporal, se sobreentiende que si no lo lleva la oración es de presente. Cfr. Walter REDmond: Lógica simbólica para todos, Xalapa: Universidad Veracruzana 1999 , cap. 7.
} 


\section{Los cuadrados modales en Tomás de Mercado}

\subsection{El antecedente medieval}

Una oración modal es aquella donde aparece un modo, una expresión como "Posible", "Necesario", "Imposible" y "Contingente" y puede ser de dos clases: de dicto o compuesta y de re o divisa. La diferencia entre ellas es la ubicación del modo: en un extremo, ya sea sujeto o predicado en las compuestas y al interior de la oración en las divisas, por ejemplo: "que Pedro dispute es posible" y "Pedro posiblemente disputa". Las divisas admiten cuantificación, como veremos.

Mercado ofrece dos cuadrados, uno para las modales compuestas y otro para las divisas. El cuadrado para las oraciones compuestas es más sencillo que el de las divisas, así que comenzaremos presentando el cuadrado de las modales compuestas haciendo notar, de paso, algunas diferencias con la tradición medieval.

El cuadrado modal para las compuestas lo encontramos ya en Pedro Hispano, William de Sherwood y Tomás de Aquino entre otros ${ }^{5}$. Son los realistas del siglo XIII y cuya doctrina lógica, especialmente la de Pedro Hispano, será "corregida y aumentada" por los autores del siglo XIV, nominalistas en buena parte, adaptándolo para que cuadrara con sus preferencias filosóficas. El cuadrado fue expresado atendiendo a las relaciones modales y sus equivalencias sin recurrir a una oración sino al "ser" sin más, según veremos, si bien recurren a oraciones en la explicación de la doctrina. Antes de proseguir diremos que el cuadrado tiene los mismos extremos que el cuadrado de las oraciones categóricas cuantificadas: $A, E, I$ y $O$ estando el modo "necesario" en la parte de arriba, que corresponde a las universales y el modo "posible" en la parte inferior correspondiente a las particulares; del lado izquierdo las afirma-

\footnotetext{
${ }^{5}$ Cfr. Pedro Hispano: Tractatus, llamados después Summule logicales, trad. de M. Beuchot, México: UNAM 1986; William de SHERWOOD: Introductiones in Logicam, ed. de Hartmut Brands y Christoph Kann, Hamburgo: Felix Meiner Verlag 1995; Tomás de Aquino: "De propositionibus modalibus", en Opuscula philosophica, edición de Raimundo Spiazzi, Turín-Roma: 1954.
} 
tivas, según las primeras vocales de la palabra affirmo y del lado derecho las negativas, según las primeras vocales de nego.

La primera diferencia es pues, la formulación de las equivalencias. A cada letra del cuadrado corresponden cuatro oraciones modales, todas equivalentes entre sí. Así que el cuadrado consta de dieciséis componentes, cuatro en cada extremo:

$A$

pur non possibile est non esse

pu non contingens est non esse re impossibile est non esse a necesse est esse

a impossibile est esse ma contingens est esse bi non impossibile est esse mus non necesse est non esse

\author{
$E$ \\ $i$ non possibile est esse \\ li non contingens est esse \\ a impossibile est esse \\ ce necesse est non esse

\section{O} \\ e possibile est non esse \\ den contingens est non esse \\ tu non impossibile est non esse \\ li necesse est esse
}

Figura 4: Cuadro modal medieval.

Aunque autores nominalistas ya habían señalado la diferencia entre contingencia y posibilidad, Tomás de Mercado conserva la contingencia como equivalente a la posibilidad dentro del cuadrado, no sin observar que "En esta acepción (a mi juicio) introducen bastante violentamente al término, y lo hacen convertible con lo posible" (p. 278). Esta es la segunda diferencia respecto a los autores del siglo XIII. Y para no dar la impresión de que en estos la doctrina es homogénea diremos que en Tomás de Aquino encontramos palabras mnemotécnicas que no aparecen en Sherwood ni en Hispano, para recordarnos la combinación de la negación al interior de cada oración. Son las sílabas que aparecen flanqueando cada oración del cuadrado anterior: $I$ a-ma-bi-mus, $O$ e-dentu-li, E i-li-a-ce y $A$ pur-pu-re-a. La clave es la presencia de las vocales, pues indican varias cosas, a: no hay negación, e: hay negación después del modo, $i$ : hay negación antes del modo, $u$ : hay negación antes y después del modo. En los novohispanos tendremos la negación aplicada al modo y al dictum, la oración calificada por uno de los cuatro modos. 


\subsection{El cuadrado para las modales compuestas}

El cuadrado modal para las modales compuestas corresponde al medieval, pero Mercado utiliza una oración, "Pedro disputa", para ejemplificarlo. La oración "Pedro disputa" es un extremo de toda la oración, digamos el extremo sujeto, y el modo es el extremo predicado, y esta combinación nos resulta en la oración modal "que Pedro disputa es necesario", y así para cada modo y sus equivalentes. Utilizaremos una constante oracional $D$ y los símbolos $\square$ y $\diamond$ para necesidad y posibilidad, una tilde $\neg$ para la negación. La imposibilidad puede definirse en términos de negación y posibilidad; la contingencia, como dice Mercado, en términos de conjunción, negación y posibilidad ${ }^{6}$. Ejemplificamos el cuadrado omitiendo la imposibilidad y la contingencia pues, como dijimos, son definibles y por tanto no son operadores primitivos. Colocamos las equivalencias entre posibilidad y necesidad en cada extremo del cuadrado.

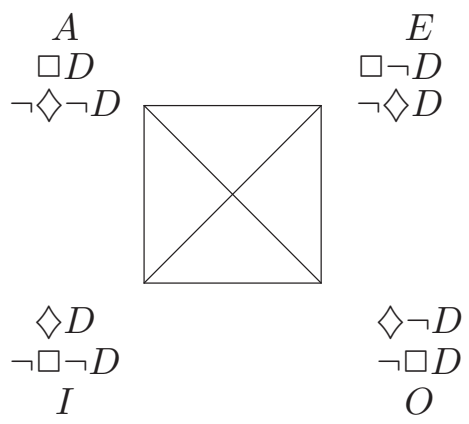

Figura 5: Cuadro de modales compuestas.

Este cuadrado modal sigue las reglas del cuadrado tradicional y sus equivalencias y oposiciones: contrarias $(A-E)$, contradictorias $(A-O, E$ $I)$, subcontrarias $(I-O)$ y subalternas $(A-I, E-O)$, equivalencias $(A, \neg O$;

\footnotetext{
“"[A]quello que puede ser y no ser, como el que yo escriba mañana”, ibidem. Se entiende: mañana puedo escribir y puedo no escribir, ambas posibilidades, por eso se le llama también doble posibilidad. Corresponde a la conjunción de las subcontrarias.
} 
$\neg A, O),(E, \neg I ; \neg E, I)$ por mencionar solo algunas, de donde puede salir un considerable número de teoremas modales. Mercado no ofrece un cuadrado modal para oraciones modales singulares divisas, donde el modo está en medio de la oración (tampoco tenemos un simbolismo adecuado en la lógica ordinaria) aunque Alonso de la Veracruz diga en algún momento que las modales divisas y compuestas sin cuantificar, esto es, con sujeto singular, son equivalentes ${ }^{7}$. Esta es quizá la razón por la que Mercado no expone el cuadrado para modales divisas, pues sería equivalente al de las modales compuestas.

\subsection{El cuadrado para las modales divisas}

\subsubsection{Explicación de los componentes del cuadrado}

Este cuadrado es más complejo pues consta de ocho entradas en lugar de cuatro, combinando las relaciones del cuadrado de oposición y equivalencia según los cuantificadores y según los modos. Trataré de explicarlo paso a paso comenzando con una oración típica del cuadrado — más bien octágono- para mostrar sus subalternas.

Recordemos dos cosas: el cuantificador universal implica al particular y no viceversa, el modo necesario implica al modo posible y no viceversa. Cada oración tendrá un cuantificador y un modo, en ese orden; el modo tendrá forma adverbial.

Usaré cursivas para indicar cuantificador y modo, negritas para indicar la relación de subalternación respecto a la oración anterior. Las relaciones serán tanto por el cuantificador como por el modo. Llamaremos universales por el cuantificador cuando tengamos la expresiones "todo", "ninguno" y universales por el modo cuando tengamos "necesariamente" e "imposiblemente" (o sus equivalentes). Particulares por el

\footnotetext{
${ }^{7}$ Cfr. Juan M. CAmpos: "Metafísica y modalidad en Fray Alonso de la Veracruz", Revista de Filosofía 28 (1998). La discusión contemporánea gira en torno a las equivalencias entre modales compuestas y divisas cuantificadas, en las llamadas fórmulas Barcan. Esto ya fue tratado por los medievales del siglo catorce. Las fórmulas Barcan son fórmulas un poco menos complejas que las que veremos en el apartado siguiente y ambas, las Barcan y las del cuadrado complejo cuantificacional y modal involucran, dicho sea de paso, el sistema modal $S 5$ de Lewis.
} 
cuantificador cuando aparezcan las expresiones "algún”, "algún... no" y particulares por el modo cuanto tengamos "posiblemente" y "posiblemente... no" (o sus equivalentes).

Permítame el lector la siguiente estrategia expositiva: usaré dos letras para abreviar cada oración. Las letras serán las vocales usuales $(A, E, I$, $O)$ pero combinadas, la primera para cuantificadores y la segunda para modos, en este orden precisamente.

\subsubsection{Las oraciones subalternas y disparatadas}

Comencemos con las afirmativas; partamos de la siguiente oración, universal tanto por el cuantificador como por el modo

\section{$A A$ : Todo hombre necesariamente disputa}

y como lo universal implica lo particular, resulta la oración subalterna por el modo, lo que expresamos en negritas

AI: Todo hombre posiblemente disputa y si la subalternación se da por el cuantificador tenemos la oración

I A: Algún hombre necesariamente disputa y tanto por el cuantificador como por el modo tendremos

\section{II: Algún hombre posiblemente disputa.}

Hemos partido de oraciones universales $(A A)$ y llegado a oraciones particulares $(I I)$ de tal manera que parece que hemos descendido de universal a particular. En efecto, la relación ha sido la subalternación, pues

$(A A)$ implica $(A I)$

$(A A)$ implica $(I A)$

$(A A)$ implica $(I I)$ 
Pero quiero prevenir al lector de una ilusión (lógica), pues podríamos pensar que todas las relaciones expresadas en la oraciones son subalternaciones, como si hubiésemos pasado gradualmente de mayor a menor, lo cual no es el caso. Considere la relación entre las oraciones

\section{AI: Todo hombre posiblemente disputa.}

\section{A: Algún hombre necesariamente disputa.}

Si bien por razón del cuantificador hay subalternación, ya que de lo universal (todo) se sigue lo particular (algún), por razón del modo no puede seguirse, pues de lo particular (posiblemente) no se sigue lo universal (necesariamente). Las oraciones $(A I)$ e $(I A)$ no son subalternas, tampoco son contrarias, ni contradictorias ni mucho menos equivalentes, pero están en el cuadrado. ¿Qué son entonces? ¿Cómo tienen cabida dentro del cuadro de oposición?

Tenemos pues una relación que no había estado presente en el cuadrado tradicional de oposición y equivalencia aristotélico y que está presente en el cuadrado modal para las divisas. Los medievales le llamaban disparatae, separadas, lógicamente independientes. Se trata de una relación simétrica. Forman parte del cuadrado complejo de tal manera que éste no puede reconstruirse en términos ordinarios, es decir, excluyendo las oraciones separadas.

Con estas cuatro oraciones $(A A, A I, I A$ e $I I)$ tenemos un lado del cuadrado, el correspondiente a las oraciones afirmativas, tanto por el cuantificador como por el modo.

Vayamos a las negativas para completar el cuadrado. Comenzamos con la universal negativa:

\section{$A E$ : Todo hombre imposiblemente disputa.}

Y antes de continuar permítame el lector una breve digresión. Puede preguntarse por qué no escribimos $(E E)$ si se trata de la oración universal negativa (tanto por el modo como por el cuantificador, como tendría que ser puesto que ya hemos expresado las oraciones afirmativas y la primera corresponde a la universal afirmativa (tipo $A A$ ). La universal negativa debería ser $(E E)$. Procedamos a seguir esta sugerencia: 
$(E E)$ : Ningún hombre imposiblemente disputa

ahora bien, decir que "Ningún hombre disputa" es afirmar que de todos los hombres, ninguno tiene la propiedad de disputar, que puede expresarse también, aunque quizá no elegantemente, como "todo hombre no disputa". Si colocamos el modo "imposiblemente" tendremos la oración $(E E)$, y si la analizamos con cuidado veremos que puede expresarse como

Todo hombre no imposiblemente disputa.

La presencia de dos negaciones juntas, expresadas arriba en negritas, altera el modo, pues al eliminarse queda posiblemente. Así

Todo hombre posiblemente disputa

y se trata de la oración $(A I)$, la segunda de nuestras primeras cuatro oraciones. Por eso la manera adecuada de expresarle es $(A E)$. Volvamos pues a la oración

$A E$ : Todo hombre imposiblemente disputa

y equivale a

Todo hombre necesariamente no disputa que nos ayuda a apreciar mejor su subalterna por razón del modo $A O$ : Todo hombre posiblemente no disputa.

La subalterna por razón del cuantificador es

IE: Algún hombre imposiblemente disputa que equivale a

Algún hombre necesariamente no disputa. 
¿Por qué no puede expresarse como $(O E)$ esta oración, pues corresponde a la particular negativa? Porque si comenzamos con $O$ tendríamos la oración

\section{Algún hombre no disputa}

y al colocar el modo al interior de la oración tenemos "algún hombre no imposiblemente disputa", donde al eliminar la doble negación (como en el caso $E E$ ) resulta "algún hombre posiblemente disputa", nuestra oración $(I I)$ de arriba.

La subalterna de $(I E)$ por razón del modo resulta en

\section{IO: Algún hombre posiblemente no disputa.}

Y con esto tenemos ya el cuadro completo, todas las oraciones que lo componen, lo hemos presentado de acuerdo a las relaciones de subalternación, que están indicadas en las flechas; las oraciones independientes constituyen el cuadrado interno, indicado por la flecha doble. Los números afuera indican las líneas a las que nos referiremos más abajo. No están expresadas otras relaciones que veremos adelante.

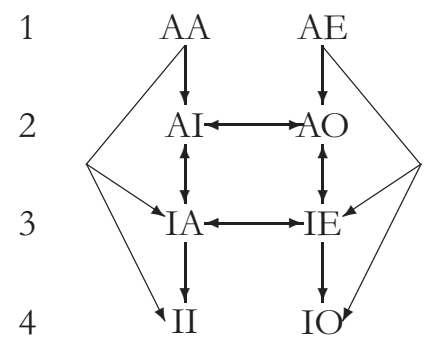

Figura 6: Esquema del octágono.

\subsubsection{Los cuadrados al interior del cuadrado}

De hecho hay que notar la presencia de seis cuadrados. Tenemos arriba dos columnas, cada una de cuatro oraciones: las superiores, en la 
línea 1 son universales y las inferiores en la línea 4 particulares siendo las líneas de en medio 2 y 3 una combinación de ambas.

Las líneas 1 y 4 forman un cuadrado externo donde las relaciones se dan simpliciter, completa o absolutamente, tanto por el cuantificador como por el modo: contrarias, subcontrarias, subalternas y contradictorias.

$1 \mathrm{AA}$

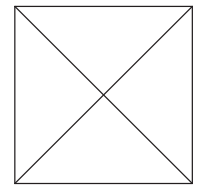

4 II
$\mathrm{AE}$

IO

Figura 7: Cuadro externo.

Las líneas 1 y 2 forman otro cuadrado donde las contrarias ocupan el "lugar" de las contradictorias $(A A, A O)$ y $(A E, A I)$; son pues contrarias por el cuantificador los extremos diagonales, siendo las oraciones de la línea $2(A I, A O)$ independientes — en los siguientes cuadrados la flecha doble indica la independencia lógica:



Figura 8: Subalternas, contrarias e independientes. 
Las líneas 2 y 3 forman otro cuadrado donde la relación de contradicción de da simpliciter, en su lugar natural en los extremos diagonales $(A I, I E)$ y $(A O, I A)$; las demás oraciones son independientes $(A I$, $A O),(A I, I A),(A O, I E),(I A, I E)$ :

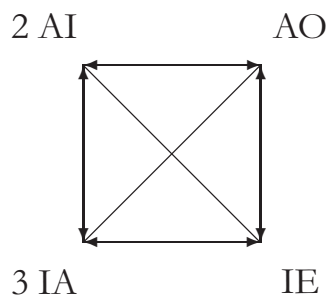

Figura 9: Contradictorias e independientes.

Las líneas 3 y 4 forman otro cuadrado donde la subalternación se da en razón del modo, las subcontrarias por razón del cuantificador pero ocupando el lugar de las contradictorias en extremos diagonales $(I A$, $I O)$ y $(I E, I I)$.

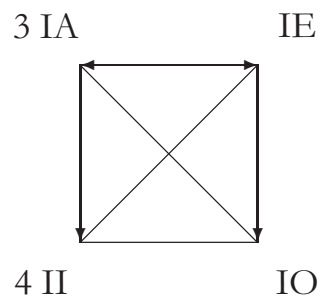

Figura 10: Subalternas, subcontrarias e independientes.

Las líneas 1 y 3 muestras las relaciones de subalternación por parte del cuantificador y son contrarias por el modo, ocupando las contrarias el lugar de las contradictorias, los extremos diagonales $(A A, I E)$ y $(A E$, $I A)$. 
$1 \mathrm{AA}$

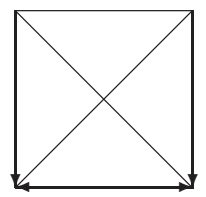

3 IA
$\mathrm{AE}$

IE

Figura 11: Subalternas, contrarias e independientes.

Las líneas 2 y 4 muestran la subalternación por parte del cuantificador y son subcontrarias por parte del modo, ocupando el lugar de las contradictorias, los extremos diagonales $(A I, I O)$ y $(A O, I I)$.



Figura 12: Subalternas, subcontrarias e independientes.

\subsubsection{El cuadrado completo}

Hemos "descompuesto" el cuadrado complejo de oposición en seis cuadrados no ordinarios. No son ordinarios pues las relaciones de contradicción, que usualmente son los extremos diagonales, aparecen "suplantadas", por decirlo así, por otras relaciones, las de contrariedad y subcontrariedad. Hemos encontrado además otra relación, la de independencia lógica que no aparece en el cuadrado de Aristóteles. El lector puede juzgar el alcance de este cuadrado novohispano y sus innovaciones. 
Todas estas relaciones combinadas en un solo cuadrado lucen así, como aparece en la traducción de Beuchot del texto de Mercado (p. 283). Donde dice "por razón del sujeto" entiéndase "por razón del cuantificador". Se usa "sujeto" porque el cuantificador califica, modifica si se quiere, el sujeto gramatical de la oración. De hecho el cuantificador rige toda la expresión, pues el modo está en medio de la oración, como corresponde a las modales divisas; "el hombre..." ha de entenderse como "algún hombre...", pues corresponde a la cuantificación particular.

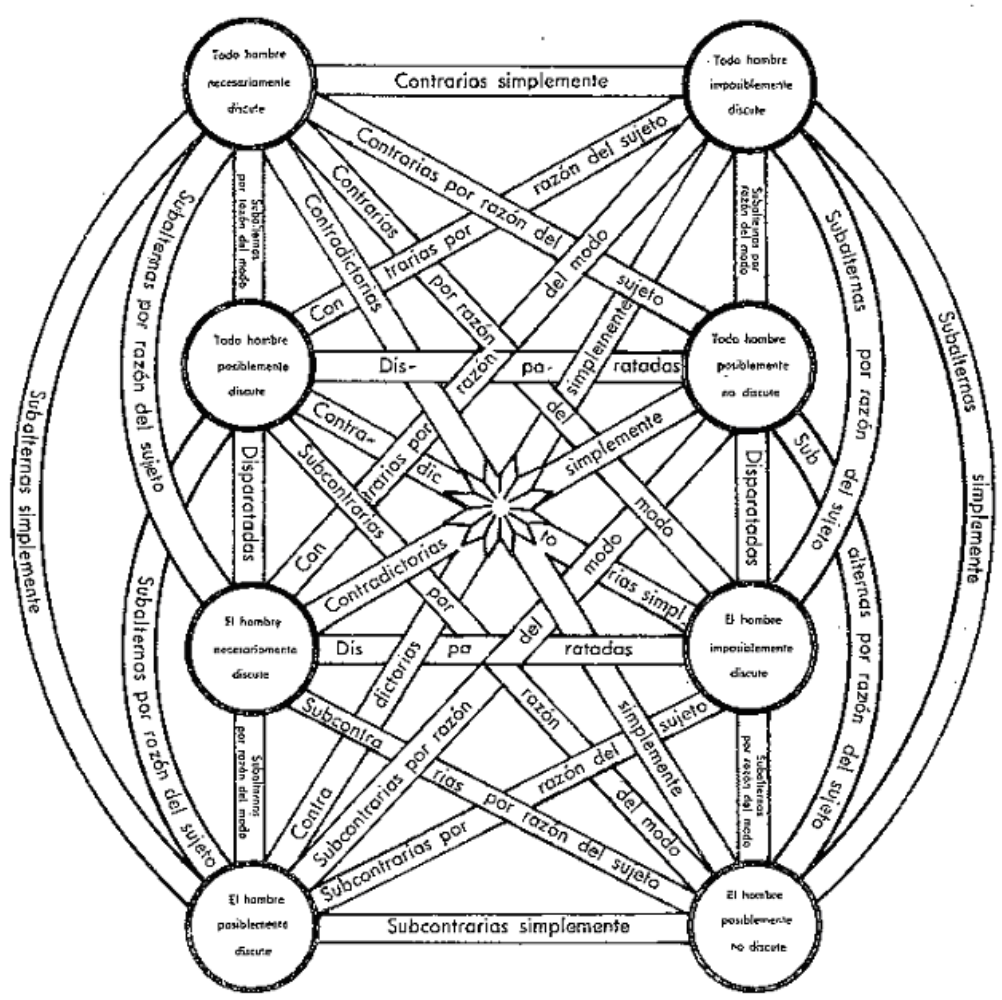

Figura magna: Octágono novohispano. 


\section{Consideraciones finales}

El cuadrado, o mejor dicho, los "cuadrados", pueden considerarse como una de las más grandes expresiones del pensamiento lógico y contienen una riqueza insospechada. Es una expresión gráfica, visible, de relaciones altamente abstractas; bastaría con desentrañar los teoremas que encierran para tener una idea de ello, y el número de teoremas puede sorprender a más de un lector ${ }^{8}$.

El cuadrado puede aplicarse a la lógica proposicional, pero también a la modal y temporal como hemos visto. En efecto, las nociones modales se expresan también en el cuadrado, así como también las nociones temporales; pueden combinarse, como lo hemos visto en el octágono que combina la modalidad y la cuantificación y que incluye las conectivas oracionales. Son "extensiones" de la lógica, pues además de las conectivas y cuantificadores asimilan otros operadores que también se rigen por las leyes del cuadrado tradicional. En esto radica su valor sintáctico, que es el que hemos expuesto; los aspectos semánticos y pragmáticos merecen un estudio aparte pues constituyen temas de la filosofía de la lógica. En nuestros días se ofrecen la mayoría de las veces en textos especiales, para cursos superiores de lógica, no en los textos para principiantes. Hay que decir, no obstante, que están presentes en la obra de los novohispanos, en sus textos que ofrecían a sus estudiantes.

Hemos mostrado una "expansión", por decirlo así, del cuadrado tradicional de Aristóteles, una aplicación a varios aspectos que son importantes e interesantes para la filosofía.

Falta mucho por estudiar, pues el cuadrado ha resultado fecundo para otros terrenos: la ética, la epistemología, la mereología, por mencionar solo algunos. Todos ellos encuentran expresión a través del cuadrado, guardando las diferencias, que las hay. Así pues el cuadrado ha mostrado ser más que un instrumento de la lógica; puede expresar el aspecto lógico de muchas "realidades" o aspectos de la realidad: el ético, el epistémico, el temporal. En efecto, la lógica de oraciones, la lógica de predicados y

\footnotetext{
${ }^{8}$ Para una idea de los teoremas del cuadrado modal sin cuantificar véase mi artículo "El cuadrado medieval de oposición proposicional y modal", Revista Española de Filosofía Medieval, 14 (2007).
} 
las extensiones de la lógica pueden todas ellas expresarse a través del cuadrado, y es lo que nos autoriza a llamarles "lógica": lógica modal, lógica temporal. Los novohispanos ya tenían presente esto en sus obras.

Por eso es importante volver a nuestros autores novohispanos que enseñaron lógica en nuestras tierras. El estudioso podrá encontrar muchas cosas de provecho; valga este breve artículo como una invitación al estudio de los novohispanos, algo podrán enseñarnos. 
